\title{
Visual Analytics for Pattern Discovery in Home Care
}

\section{Clinical Relevance for Quality Improvement}

Kavita Radhakrishnan'; Karen A. Monsen²; Sung-Heui Bae ${ }^{3}$; Wenhui Zhang'

${ }^{1}$ School of Nursing, University of Texas - Austin, Austin, TX;

2University of Minnesota School of Nursing, Minneapolis, MN;

${ }^{3}$ Ewha Womans University, College of Nursing, Seoul, Republic of Korea

\section{Keywords}

Visualization, home health nursing, Omaha System, urinary incontinence, patient outcomes

\section{Summary}

Background: Visualization can reduce the cognitive load of information, allowing users to easily interpret and assess large amounts of data. The purpose of our study was to examine home health data using visual analysis techniques to discover clinically salient associations between patient characteristics with problem-oriented health outcomes of older adult home health patients during the home health service period.

Methods: Knowledge, Behavior and Status ratings at discharge as well as change from admission to discharge that was coded using the Omaha System was collected from a dataset on 988 deidentified patient data from 15 home health agencies. SPSS Visualization Designer v1.0 was used to visually analyze patterns between independent and outcome variables using heat maps and histograms. Visualizations suggesting clinical salience were tested for significance using correlation analysis.

Results: The mean age of the patients was 80 years, with the majority female (66\%). Of the 150 visualizations, 69 potentially meaningful patterns were statistically evaluated through bivariate associations, revealing 21 significant associations. Further, 14 associations between episode length and Charlson co-morbidity index mainly with urinary related diagnoses and problems remained significant after adjustment analyses. Through visual analysis, the adverse association of the longer home health episode length and higher Charlson co-morbidity index with behavior or status outcomes for patients with impaired urinary function was revealed.

Conclusions: We have demonstrated the use of visual analysis to discover novel patterns that described high-needs subgroups among the older home health patient population. The effective presentation of these data patterns can allow clinicians to identify areas of patient improvement, and time periods that are most effective for implementing home health interventions to improve patient outcomes.

\section{Correspondence to:}

Kavita Radhakrishnan, PhD RN MSEE

Assistant Professor

School of Nursing

University of Texas - Austin

1710 Red River Street,

Austin, TX 78701-1499

UNITED STATES

Tel: (512) 471-7936

Fax: (512) $471-3688$

Email: kradhakrishnan@mail.nur.utexas.edu
Appl Clin Inform 2016; 7: 711-730

http://dx.doi.org/10.4338/ACl-2016-03-RA-0049

received: 6. April 6, 2016

accepted: June 23, 2016

published: July 27, 2016

Citation: Radhakrishnan K, Monsen KA, Bae S-H, Zhang W. Visual analytics for pattern discovery in home care: Clinical relevance for quality improvement. Appl Clin Inform 2016; 7: 711-730

http://dx.doi.org/10.4338/ACl-2016-03-RA-0049 Funding

The University of Texas Austin New Faculty Start-up Funds for Dr. Radhakrishnan. 


\section{Introduction}

Home health, consisting of agencies or organizations that primarily provide skilled nursing and other therapeutic services to patients in their homes, is one of the fastest growing health care sectors $[1,2]$. In 2012, the Centers for Medicare \& Medicaid Services (CMS) reported that such agencies were serving approximately 4.5 million Medicare and Medicaid patients annually. However, home health agencies face challenges, including CMS decreases in payments for home health services [3] and increases in accountability for patient outcomes. If home health nurses are to provide efficient, effective care to home health patients with chronic diseases, they must be given innovative ways to identify characteristics of patients, that are associated with patients' health outcomes. Knowledge of such associations can inform decision making and allocation of resources, improve home health clinicians' ability to avoid adverse health outcomes, and help determine which home health interventions are appropriate for specific home health subpopulations [4].

The Institute of Medicine and others now encourage documentation of psychosocial variables that include the social determinants of health $[5,6]$. Datasets that capture data on psychosocial variables include the Omaha System [7], a taxonomic health care terminology, and the Medicare-mandated Outcome and Assessment Information Set (OASIS), both of which are used by home health nurses to manage comprehensive, holistic clinical information about their older adult patients in home health electronic health records (EHRs). However, multidimensional EHR patient data are generally presented in numbers and tables, which increases clinicians' cognitive load and makes it time-consuming to extract and classify meaningful information both in practice and in research. Because practicing home health nurses cannot retrieve and interpret multidimensional patient data easily, they cannot review and utilize such information effectively or efficiently for making decisions about the delivery of care. Data visualization can inform clinicians' decisions about quality of care, influencing not only how quickly clinicians interpret information, but also how they interpret it [8]. Patient data and nursing care documented with the Omaha System and OASIS need to be integrated and presented so that one can easily understand and evaluate the interplay of multiple variables [9].

In research, visual analysis is an innovative, emerging technique that can reduce the cognitive load of information, allowing users to easily interpret and assess large amounts of data. Visualization techniques enable the extraction of key information, making visual analysis a powerful means with which to understand and examine large quantities of data [10]. A plethora of visualization techniques such as heat maps, histograms, radial displays, or line graphs are available to visualize large clinical datasets depending on the purpose of the visualization and the complexity of the data being displayed [10]. In a study that evaluated speed and quality of assessment by medical students by comparing three line graphs and a table method for visualizing lab results, different visualization methods were preferred based on the complexity of the data being presented [11]. However, identifying meaningful patterns in visualizations of large EHR-related datasets remains a challenge due to the size and complexity of EHR data [12]. Recent studies have demonstrated the use of data visualization techniques such as steam graph, heat maps and sunburst graphs to identify hidden patterns in Omaha System data documented by public health nurses [8, 13-14]. No studies, however, have tried to visualize the association of home health patient with problem-oriented patient outcomes during the home health service period.

\section{Objectives}

The purpose of our study was to examine home health data using visual analysis techniques to discover clinically salient associations between patient characteristics with problem-oriented health outcomes of older adult home health patients during the home health service period.

\section{Methods}

Review by The University of Texas - Austin Institutional Review Board found that this study did not require determination as human subjects research. The study used an existing dataset for which the 
data were abstracted from clinical documentation on 988 de-identified Medicare home health patients at admission and discharge from 15 home health agencies during the year 2004 [15]. All patients were represented by an unique fictitious identifier. Data obtained with the Omaha System Problem Classification Scheme and Problem Rating Scale for Outcomes as well as OASIS data on patient demographics (e.g., age), physiological characteristics (e.g., primary and co-morbid diagnoses), home health service characteristics (e.g., length of stay), and discharge disposition were used in the study.

\subsection{The Omaha System data}

The Omaha System is a taxonomic health care terminology and measure that enables the management of comprehensive, holistic clinical information in health care [7]. While Omaha System is not currently a mandated standard of clinical data collection, it has been used widely in community settings globally, especially in public health and home care, in order to describe and measure the impact of nursing and healthcare services on patient care and outcomes [7, 16,17]. The Omaha System comprises an assessment component (Problem Classification Scheme), a care plan/services component (Intervention Scheme), and an evaluation component (Problem Rating Scale for Outcomes) [16]. The Problem Classification Scheme comprises 42 problems or concerns that fall within four domains that represent patients' physiological and psychosocial health within the environment, along with personal health choices expressed by health-related behaviors [7]. The Problem Rating Scale for Outcomes comprises three five-point Likert-type ordinal measures that rate severity in a descending order ( 1 - worst outcome, 5 - best outcome) of patients' problem-specific knowledge $(\mathrm{K})$, behavior (B), and status (S). Knowledge is defined as what the client knows, Behavior as what the client does, and Status as the number and severity of the client's signs and symptoms or predicament [7].

\subsection{Outcome and Assessment Intervention Set (OASIS)}

The Medicare dataset OASIS was designed to standardize data collection for monitoring outcomes, clinical assessment, and care planning, as well as for evaluation of home health care service outcomes [18]. The dataset used in this study consisted of three domains from the OASIS tool: demographics, clinical status, and health service utilization [19]. OASIS data are collected by home care clinicians (RNs or therapists) at several time points during delivery of home care services. The most comprehensive assessments take place during admission (start of care) and at discharge or death, with other data collection points at 60-day intervals while patients receive home health care services, or upon transfer to another site of care (i.e., hospital, hospice, nursing home) [20].

\subsection{Cleaning and pre-processing of the dataset}

The SPSS v20 analytical suite was used to clean and prepare the dataset for visual analysis. The original dataset was organized with each Omaha System problem as the unit of analysis. For the present dataset, variables were created to represent changes in knowledge, behavior, and status scores from admission to discharge for each problem. We created a second dataset with each patient as the unit of analysis, using the Restructure feature in SPSS. For this dataset, variables were created to represent the number of problems documented for a patient, average KBS scores at discharge for all problems documented for an individual patient as well as the average difference in KBS scores for all the problems documented for a patient from admission to discharge. The average discharge KBS scores were stratified within 6 groups by rounding the values to the closest whole number $(0,1,2,3,4,5)$. The average difference in KBS scores were stratified within 6 groups based on the scores' values ( -2 to $-1.1,-1$ to $-0.1,0$ to $0.9,1$ to $1.9,2$ to $2.9,3$ to 3.9 ).

Physiological characteristics were represented by primary diagnosis, co-morbidities, and Charlson co-morbidity index. Higher Charlson index indicated higher levels of co-morbidity and illness severity. In the original dataset, the Clinical Classification System (CCS) was used to compress 51 types of primary diagnoses within 11 disease groups ( Table 1 and $>$ Supplementary Table 1 ). OASIS entries for co-morbid diagnoses that were coded using ICD-9-CM codes were then con- 
verted to dichotomous nominal variables representing 17 major disease groups ( $\$$ Supplementary Table 2) as classified in the ICD-9-CM code directory [21].

Descriptive analysis was conducted and frequencies were obtained for all the variables. Means, standard deviations, and percentiles for all continuous variables as well as frequency distributions for all discrete variables were obtained. Continuous variables of age and episode length were stratified into groups of $<75,75-84,>84$ years and $<25$ th percentile, 26 th -74 th, $>75$ th respectively for visual analyses employing histograms. Variables with $<5 \%$ variance in values were removed from further analysis because of lack of sufficiently discriminant information.

\subsection{Visual Analysis}

SPSS Visualization Designer v1.0 was used to visually analyze the associations among the variables. For the patient-level analysis, patterns of relationships between patients' demographic or physiological characteristics, home health service characteristics (length), discharge disposition, average KBS rating at discharge and average change in KBS rating from admission to discharge were explored. For the problem-level analysis, patterns of relationships between patients' demographic or physiological characteristics, home health service characteristics (length), discharge disposition, frequency of specific problems, KBS rating at discharge and changes in KBS ratings for each problem from admission to discharge were explored. Patterns in the data were visualized as relationships by using heat maps or histograms. Heat maps are particularly effective for visualizing multi-dimensional data from the present dataset as it can display a large number of rows and columns of data in a relatively compact area [22]. Color saturation in heat maps help differentiate among different groups in large multi-dimensional datasets. On the other hand, histograms in which color saturation is used for equalization are preferable to indicate the magnitude of difference among the different groups in large multi-dimensional datasets [10].

Visualization using heat maps can be described as a matrix shaded to show features arranged on the $x$-and $y$-axes $[23,24]$ ( Figure 1). For example, the key for the visualization using heat maps included representation of age, episode length or Charlson Index through color saturation, primary diagnosis or problems in columns, differences in KBS ratings over time or KBS ratings at discharge within rows. This enabled multi-dimensional exploration of the relationships among demographic, physiological, and patient outcome variables. Visualization using histograms can be described as the use of rectangles whose area is proportional to the frequency of a variable and whose width is equal to the class interval ( $>$ Figure 2). The key for the visualization using histograms included representation of problems or primary diagnoses as columns, count of problems as rows, gender as panels and difference in KBS ratings over time or KBS rating at discharge through different color hues.

We examined each visualization for potential meaning by identifying visualizations with consistent difference in patterns along the cell columns. The visualizations were examined on a laptop with lighting in the room adjusted to avoid any glare on the laptop screen. Clinically salient patterns were defined as patterns with relevance to the home health nursing practice. The first author's extensive research and clinical experience in the home health setting also informed the identification of patterns that may be clinically relevant to home health nurses. After identifying the clinically salient patterns from all the visualizations, the first author shared and discussed $20 \%$ of the number of identified clinically salient patterns with the second author, an expert in data visualization, in order to validate the identified visual patterns and suggested clinical relevance. We then tested the visualizations that suggested clinical salience for statistical significance by using correlation analysis and chi-square to conduct the bivariate comparisons. Visualizations were also selected for statistical significance testing if the count in any column or columns in a histogram appeared significantly different across the panels. Finally, we tested the identified patterns using multiple regression unadjusted and adjusted analyses of association between patient and home health service characteristics and difference in KBS ratings or KBS ratings at discharge. In the adjusted analysis, we controlled for age, gender, Charlson index and episode length. 


\section{Results}

Table 1 provides descriptive statistics for patients at both the patient and problem levels of analyses. The mean age of the patients was 80 years, with the majority female (66\%). Patients averaged four problems and 38 days as the average home health length of stay. The top primary diagnosis group comprised disorders related to the central nervous system (31\%), followed by cardiac diagnoses (15\%). The most commonly documented problems were related to Neuro-muscular function (16\%) and Pain (13\%). Overall, across all the Omaha System problems, the KBS rating of $80 \%$ of all problems were rated at 4 or 5 at discharge and $70 \%$ of all problems improved by 1 or more KBS rating from admission to discharge.

\subsection{Visualizations}

After eliminating variables with less than $5 \%$ variance, a total of 150 visualizations were conducted to explore relationships between age, gender, home health length of stay, and co-morbidity burden with KBS ratings at discharge and change in KBS rating from admission to discharge. 105 visualizations were conducted at the patient level of analysis and 45 variables were conducted at the problemlevel analysis.

Figure 1 presents an example of heat map visualizations. In $>$ Figure 1 , the heat map displays home health clients' primary diagnoses, home health length of stay, and average change in knowledge ratings for problems from admission to discharge. Increasing red hues represent longer length of stay. Improvement in knowledge from admission to discharge is shown on the $y$-axis, and primary diagnoses are represented on the $x$-axis. The visualization in $>$ Figure 1 suggests that for genito-urinary related primary diagnoses, highest improvement in knowledge is earlier in the home health episode, whereas for integumentary related primary diagnoses, highest improvement may be later in the episode. Of the two suggested clinically salient patterns, association of home health length of stay with knowledge was statistically significant for the genito-urinary related primary diagnoses only.

Figure 2 presents an example of visualization of statistically significant associations using histograms. The histograms display the patient primary diagnoses, gender, and change in status rating from admission to discharge. The histograms within the panels of males and females were compared for proportional changes in color hues to detect differences in patterns between the genders. This visualization suggests that female home health clients differed on change in status for genito-urinary and infectious disease - related primary diagnoses as compared to male home health clients.

After each visualization was examined for meaningful patterns, 69 patterns appeared to be clinically relevant. Bivariate associations were tested, revealing 21 significant associations $(p<0.05)$ as shown in the unadjusted column of $>$ Table $2 \mathrm{a}$ and $>$ Table $2 \mathrm{~b}$. Further, after adjusting for age, gender, co-morbidity burden (Charlson Index) and episode length, 14 associations related to urinary diagnoses and problems, and Pain related Omaha System problems remained significant.

\section{Discussion}

In this study, we have used visual analysis to examine home health data from 15 home health agencies to reveal clinically salient patterns, which led to hypotheses on certain patient characteristics, home health service characteristics, and health related outcomes. Further statistical techniques including adjustment analysis validated some of the hypotheses revealed through our visual analysis, particularly the Omaha System problems related to Urinary function such as urinary incontinence, and Pain.

Through visual analysis, the adverse association of longer home health episode length with worse behavior or status outcomes for patients with impaired urinary function was revealed. After adjusting for age, gender and co-morbidity burden, association of episode length remained significant for behavior outcomes for patients with impaired urinary function. Some examples of behavior outcomes related to impaired urinary function include behaviors such as timed voiding, reduce intake of caffeinated beverages, or monitor incontinence related symptoms in order to reduce the inci- 
dences of incontinence. In the United States, the financial burden presented by urinary incontinence is significantly high as the cost of bladder incontinence among adults in 2000 was estimated at $\$ 19.5$ billion, with $\$ 14.2$ billion incurred by community residents [25]. Urinary incontinence has adverse implications on quality of life, caregiving, and service delivery for older home health patients [25]. The visualization patterns suggest implications for home care practice: The window of opportunity to improve knowledge and behavior outcomes for patients with impaired urinary function is earlier in the home health episode than later. Home health clinicians can use this insight, derived through visual analysis, to frontload their interventions, use additional support resources, find creative ways to deliver patient education or engage patients to modify behaviors on urinary function earlier in the home health episode. This hypothesis should be examined in practice settings for relevance. Alternatively, the visual analysis may also suggest that impaired urinary function such as incontinence is a complex problem that may result in longer episode length. John et al., (2014) [26] found that increased severity of urinary incontinence had increased adverse association with hospital length of stay and mortality. In a survey study, home care agencies that employed wound-ostomy nurse intervention services had significantly better outcomes for home health patients with urinary incontinence [27]. Unfortunately, in the current study we do not have data on the presence of specific interventions such as wound ostomy certified nursing services to derive associations between severity and interventions on episode length for impaired urinary function.

Female gender was associated with better change in status for diagnoses related to urinary function as compared to males. This finding aligns with the literature, especially for stress incontinence issues [28]. However, data on the specific urinary function impairment was not available in our current study. This finding also suggests the importance of further investigation of older males' contextual situations - for example, to determine whether presence of caregivers is associated with improvement in older males' behavior related to urinary function. Also, the visual patterns suggest the need to explore whether current home health teaching and interventions are more effective or satisfying for females as compared to males. Gender, however, did not have any association with knowledge or behavior outcomes for patients with impaired urinary function in the current study.

The pattern identified in the histogram analysis that patient characteristics of higher age and comorbidity burden adversely related to changes in health status or behavior for endocrine-related diagnoses ( Supplementary Figure 1) and Omaha System problems of Urinary function and Pain ( Supplementary Figure 2) were validated in bivariate association analyses. This pattern is consistent with current knowledge where complications of frailty, and multiple co-morbidities were among the barriers to better diabetes [29], urinary function [28] or pain management [30, 31] outcomes as well as related behavior changes in the elderly. With the presence of complications such as immobility, interventions focusing on urinary incontinence alone will not improve behavioral outcomes. Additionally, mounting evidence suggests that pain in the population of patients with chronic illness is rarely treated or remains untreated [32]. Systemic and organizational barriers such as limited time for conduct of comprehensive assessments by clinicians, or limited reimbursement for inter-disciplinary services obstruct adequate pain management for patients with chronic illness [32]. Complicated diagnosis and treatment of concurrent problems and co-morbidities, higher rates of medication side effects, and higher rates of treatment complications along with lack of an evidence base concerning the pharmacokinetic and pharmacodynamic changes that occur with aging further impede effective pain treatment among older adults [33, 34]. Implications for home health include intensifying the role of palliative education and services to relieve pain and suffering among older home health patients with severe comorbidity burden. Older age and severity of co-morbidity burden can inform modification of current interventions or design of interventions that can enable patients with endocrine disorders, pain or impaired urinary function achieve optimal outcomes. However after adjusting for age, gender and episode length, association of co-morbidity burden (Charlson index) with behavior outcomes remained significant for Urinary Function problem alone.

Association of increased Knowledge with increased improvement in health Status and Behavior for all primary diagnoses and Omaha System problems was revealed through visual analysis. Also improvement in Behavior from admission to discharge was also associated with improvement in Status for all primary diagnoses and Omaha System problems. This finding aligns with previous studies in a completely different populations and reinforces the importance of a comprehensive in- 
tervention strategy aiming to improve overall patient health, including Knowledge, Behavior, and Status.

The study demonstrated the application of common visualization techniques of heat maps and histograms to identify patterns in home health clinical data that was collected using standardized terminologies. Our visual analysis illuminated patterns for problems or diagnoses that might be typically neglected; this information could not have been derived from a theoretical perspective alone. In this study, we used visualization techniques to identify hidden patterns within the dataset without relating patient characteristics to particular outcomes. Some of these patterns related to Urinary function, Hearing and Skin problems remained significant even after adjusting for age, gender, co-morbidity burden and episode length. As with all observational studies, visual analysis does not infer causation, however factors such as long episode length in combination with KBS scores appears indicative of the severity in the patients' health status and the patterns we identified may show associations that can be further examined for causal pathways. Also, despite the fact that impaired urinary function or pain was not in the top five diagnosis groups or Omaha System problems, urinary function and pain still showed up as conspicuous patterns in many of the visualizations. This suggests the implication of elevating the importance of urinary incontinence and pain in home health research.

\subsection{Limitations and suggestions for future research}

As with any data mining analysis, researchers who employ visual analyses can expect extensive preprocessing and cleaning to prepare a dataset for meaningful analysis. Our visualization study could have also benefitted with additional data that informed on patients' contextual environment. Availability of nursing intervention data and contextual information such as presence of caregivers might have provided an additional layer of information for visualizing meaningful patterns between patient characteristics and outcomes. In addition, possible inconsistencies in documentation of the KBS ratings across problems and clinicians [17] may have influenced the current visualization patterns.

In the future, reliability of visualizations can be enhanced through datasets with improved consistency in documentation due to additional training of clinicians or availability of charting templates. Further research is needed to validate the patterns that were found and to design interventions that address potential opportunities to improve quality of care that the patterns suggest. The visual analysis helps present data in a manner that does not require advanced statistical or theoretical knowledge on the part of consumers such as home health nurses or case managers. Even though, only about $20 \%$ (14) of the 69 potential associations identified through the visualization method were found to be significant, visualizations can still perform as a valuable tool for clinicians to confirm their hunches or intuitions about certain patient patterns. Conducting visualizations on an "as needed" basis rather than explore all potential associations could make this method more effective and practical in the home health clinicians' practice setting. Future research should also evaluate the impact of visual pattern analysis on home health clinicians' workflow and efficiency as well as their perceptions on the use of visualization to inform and prioritize their care planning for patients with different needs.

\section{Conclusion}

We have demonstrated the use of visual analysis to discover novel patterns that described highneeds subgroups among the older home health patient population. The effective presentation of these data patterns can allow clinicians to identify areas of patient improvement, and time periods that are most effective for implementing home health interventions to improve patient outcomes. 


\section{Clinical Relevance Statement}

1. Longer home health episode length and higher Charlson co-morbidity index were adversely associated with behavior or status outcomes for patients with impaired urinary function, as revealed through visual analysis.

2. Home health clinicians could frontload their interventions, use additional support resources, find creative ways to deliver patient education or engage patients to modify behaviors on urinary function earlier in the home health episode than later.

3. Severity of co-morbidity burden can inform modification of current interventions or design of interventions to enable patients with impaired urinary function achieve optimal outcomes.

4. Future research should evaluate the impact of visual pattern analysis on home health clinicians' workflow and efficiency as well as their perceptions on the use of visualization to inform and prioritize their care planning for patients with different needs.

\section{Conflicts of Interest}

The authors do not report any conflict of interest in conducting this study.

\section{Protection of Human and Animal Subjects}

The study was performed in compliance with the World Medical Association Declaration of Helsinki on Ethical Principles for Medical Research Involving Human Subjects, and was reviewed by The University of Texas - Austin Institutional Review Board. The review by The University of Texas - Austin IRB found that this study did not require determination as human subjects research.

\section{Acknowledgement}

1) This study was conducted through the University of Minnesota Omaha System Partnership Data Collaborative. The authors acknowledge Dr. Bonnie Westra, PhD, RN, FAAN, FACMI for her support and contribution.

2) The authors gratefully acknowledge the editorial services of John Bellquist, Editor, CAIN Center and the NINR funded Center for Transdisciplinary Collaborative Research in Self-management Science (TCRSS), School of Nursing, University of Texas at Austin. 


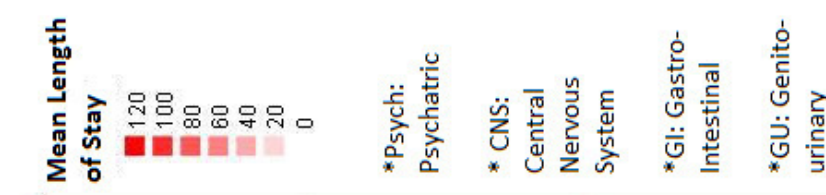

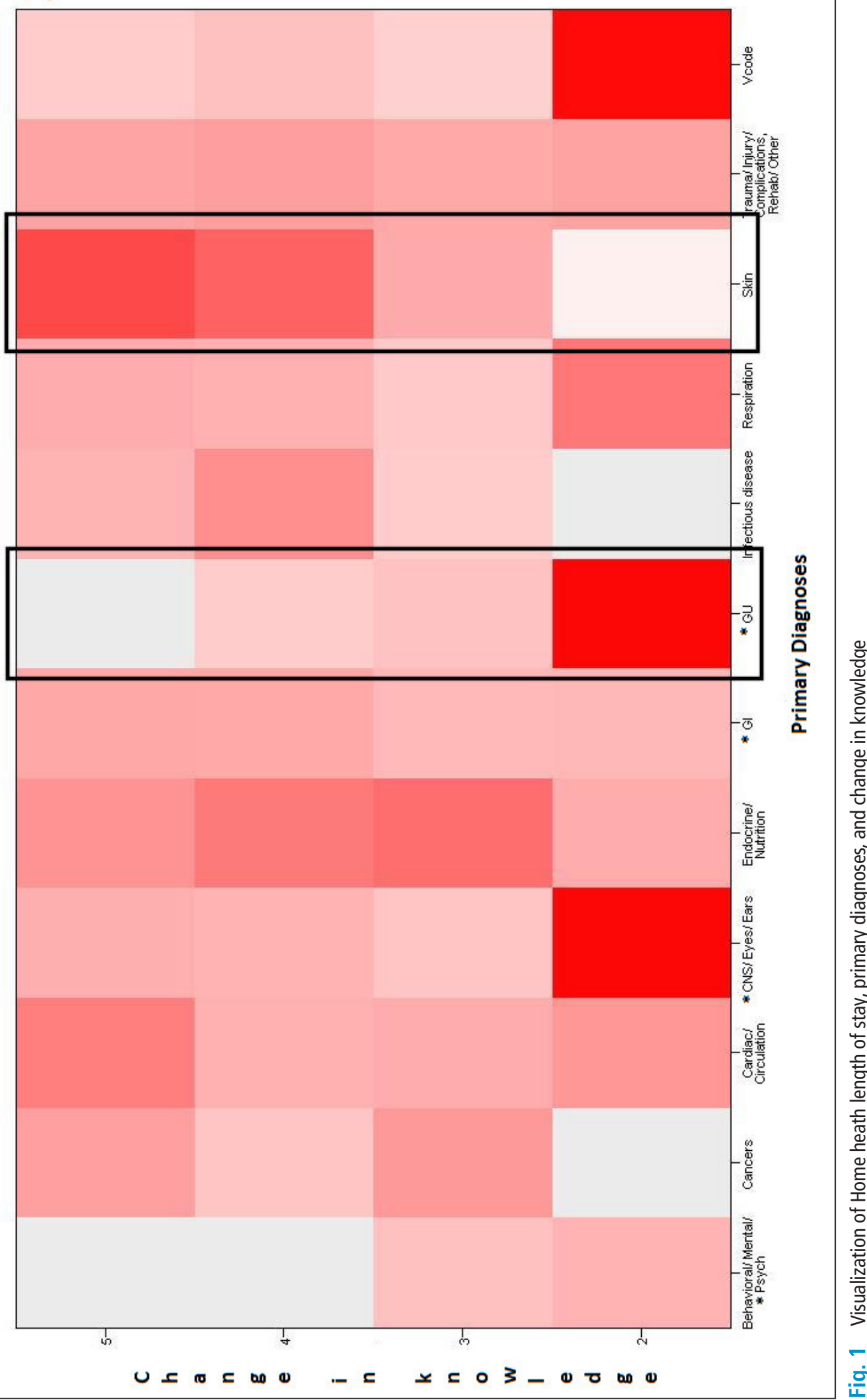



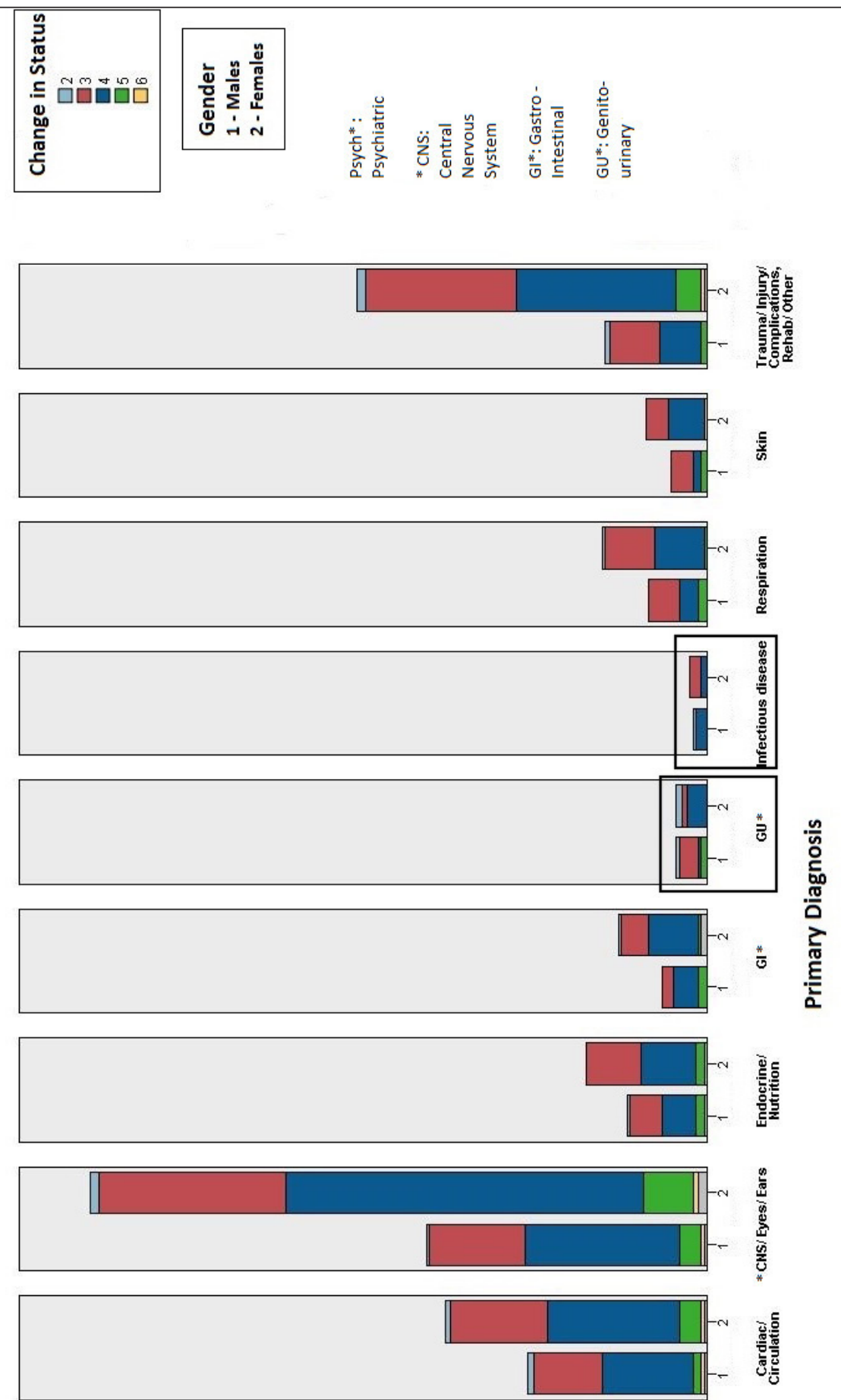

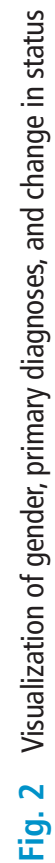




\begin{tabular}{|c|c|}
\hline \multicolumn{2}{|l|}{ Patient Characteristics } \\
\hline Age (mean, SD, in years) & $79.76 \pm 7.57$ \\
\hline Gender & n (\%) \\
\hline Male & $334(34)$ \\
\hline Female & $654(66)$ \\
\hline Race & n (\%) \\
\hline White & 976 (99) \\
\hline Not White & $12(1)$ \\
\hline Charlson Index & n (\%) \\
\hline 0 & $485(49)$ \\
\hline 1 & $269(27)$ \\
\hline 2 & $149(15)$ \\
\hline 3 & $58(6)$ \\
\hline$\geq 4$ & $29(3)$ \\
\hline Primary Diagnosis (11 CCS groups) & n (\%) \\
\hline Infectious disease & $10(1.0)$ \\
\hline Cancers & $45(4.6)$ \\
\hline Endocrine/ Nutrition & $65(6.6)$ \\
\hline Behavioral/ Mental/ Psych & $3(0.3)$ \\
\hline CNS/ Eyes/ Ears & $310(31.4)$ \\
\hline Cardiac/ Circulation & $148(15.0)$ \\
\hline Respiration & $56(5.7)$ \\
\hline GI & $43(4.4)$ \\
\hline GU & $21(2.13)$ \\
\hline Skin & $34(3.4)$ \\
\hline Trauma/ Injury/ Complications, Rehab/ Other & $156(15.8)$ \\
\hline Type of Comorbidity (Top 5) & n (\%) \\
\hline ICD 7 (Circulatory system) & $455(46.1)$ \\
\hline $\begin{array}{l}\text { ICD } 16 \text { (Symptoms, signs and ill-defined condi- } \\
\text { tions) }\end{array}$ & $389(39.4)$ \\
\hline ICD 13 (Musculoskeletal system) & $338(34.2)$ \\
\hline ICD 3 (Endocrine and immunity) & $231(23.4)$ \\
\hline ICD 8 (Respiratory system) & $132(13.4)$ \\
\hline Episode Length (mean, SD, in days) & $38.38 \pm 46.15$ \\
\hline Number of Problems (mean, SD) & $4.0 \pm 2.45$ \\
\hline Type of Problems (Top 5) & n (\%) \\
\hline Neuro-Musculoskeletal function & $697(16.2)$ \\
\hline Pain & $539(12.5)$ \\
\hline Skin / Integumentary & $518(12.0)$ \\
\hline Medication Regimen & $380(8.8)$ \\
\hline Circulation & $318(7.4)$ \\
\hline
\end{tabular}

Table 1

Patient characteristics: Descriptive statistics 


\begin{tabular}{|l|l|}
\hline Patient Characteristics & \\
\hline Knowledge Rating at Discharge & $\mathbf{n}(\%)$ \\
\hline 3 or below & $774(18.1)$ \\
\hline 4 & $2544(59.0)$ \\
\hline 5 & $952(22.1)$ \\
\hline Behavior Rating at Discharge & $\mathbf{n}(\%)$ \\
\hline 3 or below & $402(9.4)$ \\
\hline 4 & $2289(53.1)$ \\
\hline 5 & $1579(36.6)$ \\
\hline Status Rating at Discharge & $\mathbf{n}(\%)$ \\
\hline 3 or below & $753(17.6)$ \\
\hline 4 & $2276(52.8)$ \\
\hline 5 & $1241(28.8)$ \\
\hline $\begin{array}{l}\text { Difference in Knowledge from Admis- } \\
\text { sion to Discharge }\end{array}$ & $\mathbf{n}(\%)$ \\
\hline 0 or below & $1040(24.5)$ \\
\hline 1 & $2072(48.1)$ \\
\hline 2 & $951(22.1)$ \\
\hline 3 or higher & $174(4.1)$ \\
\hline Difference in Behavior from Admission & $\mathbf{n}(\%)$ \\
\hline to Discharge & $1167(27.5)$ \\
\hline or below & $2044(47.4)$ \\
\hline 1 & $953(22.1)$ \\
\hline 2 & $72(1.7)$ \\
\hline 3 or higher & $2184(50.7)$ \\
\hline Difference in Status from Admission to & $\mathbf{n}(\%)$ \\
\hline or below & $821(19.0)$ \\
\hline 1 & $105(2.5)$ \\
\hline or higher & \\
\hline
\end{tabular}

Table 1

Continued 


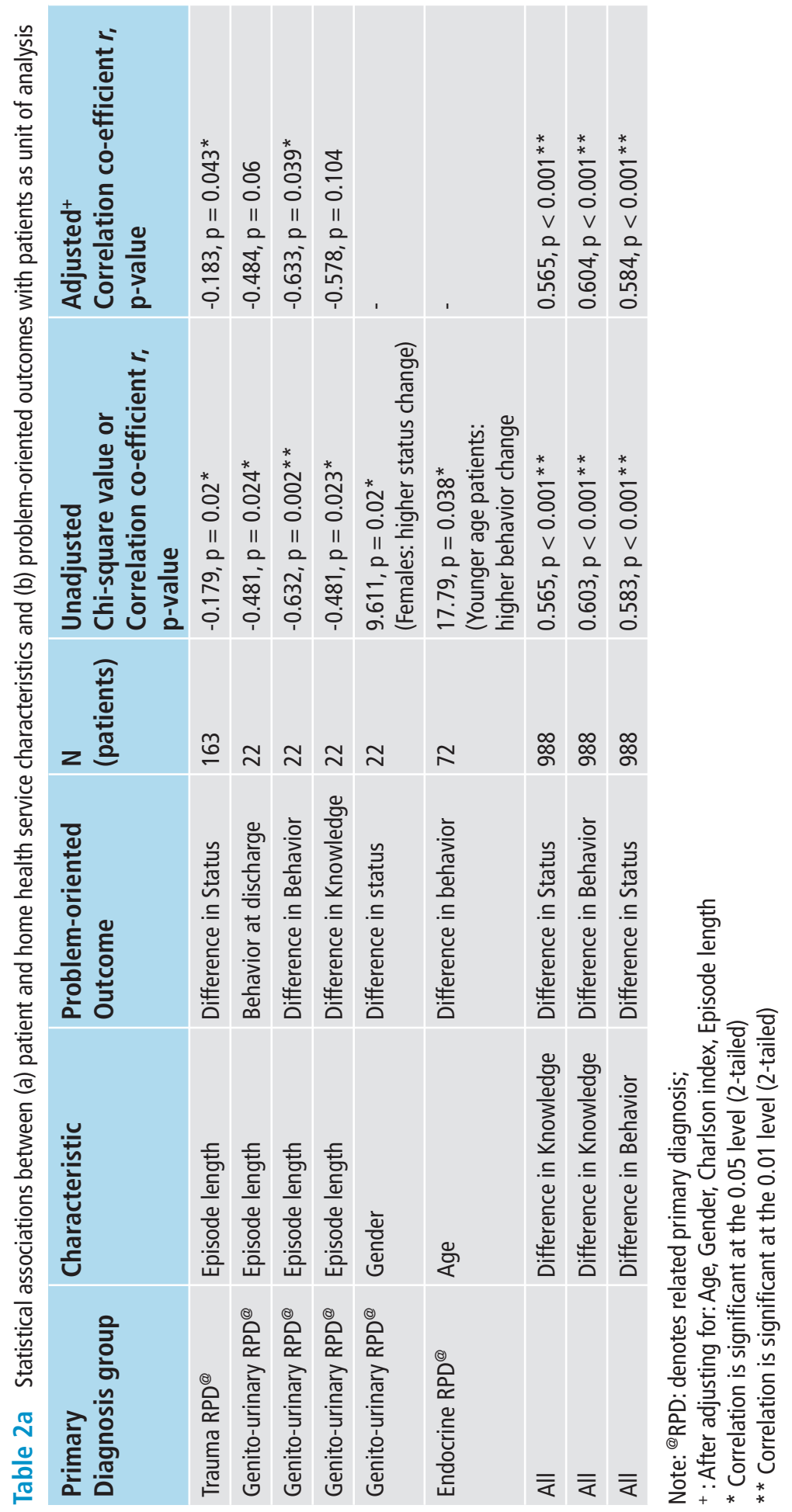




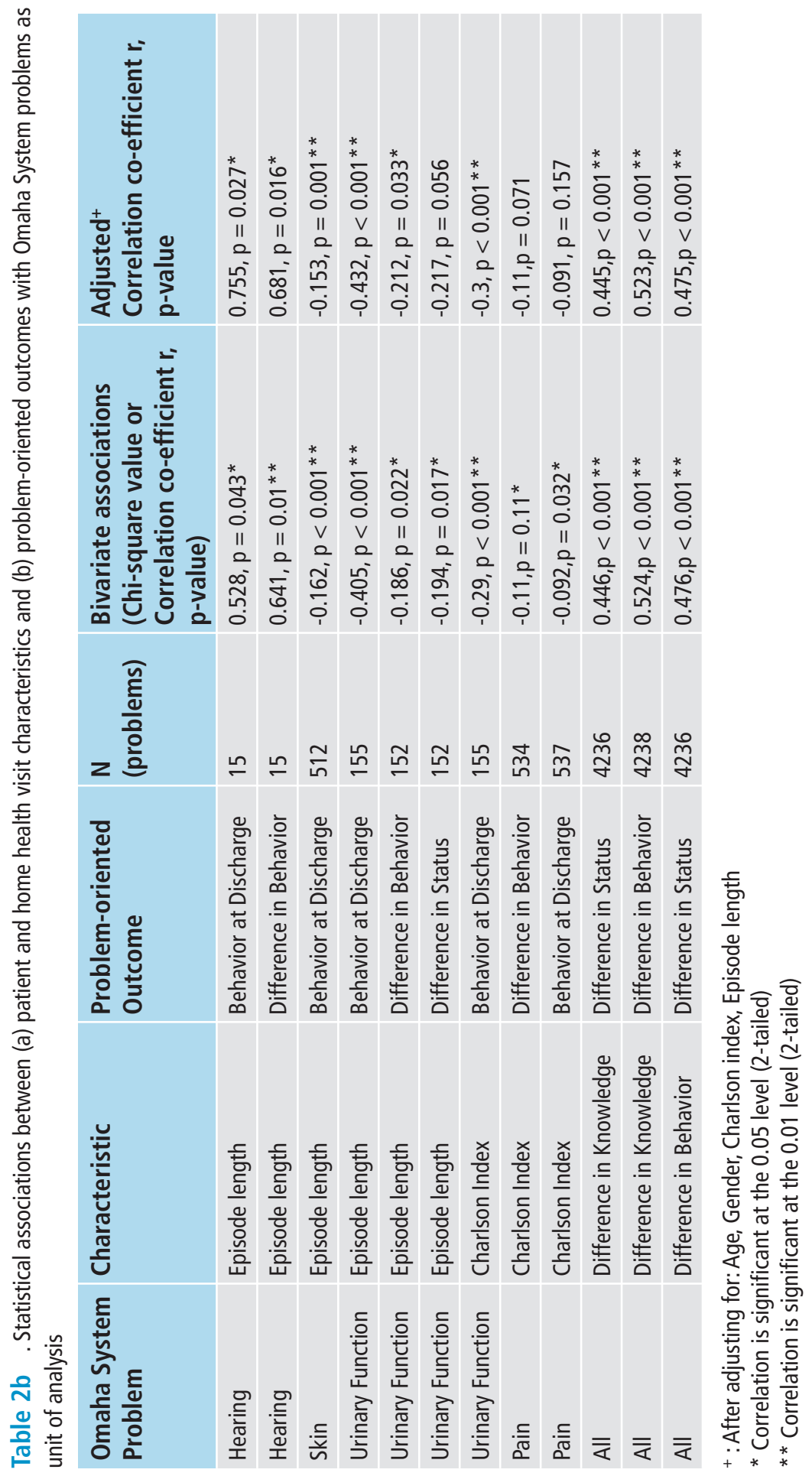




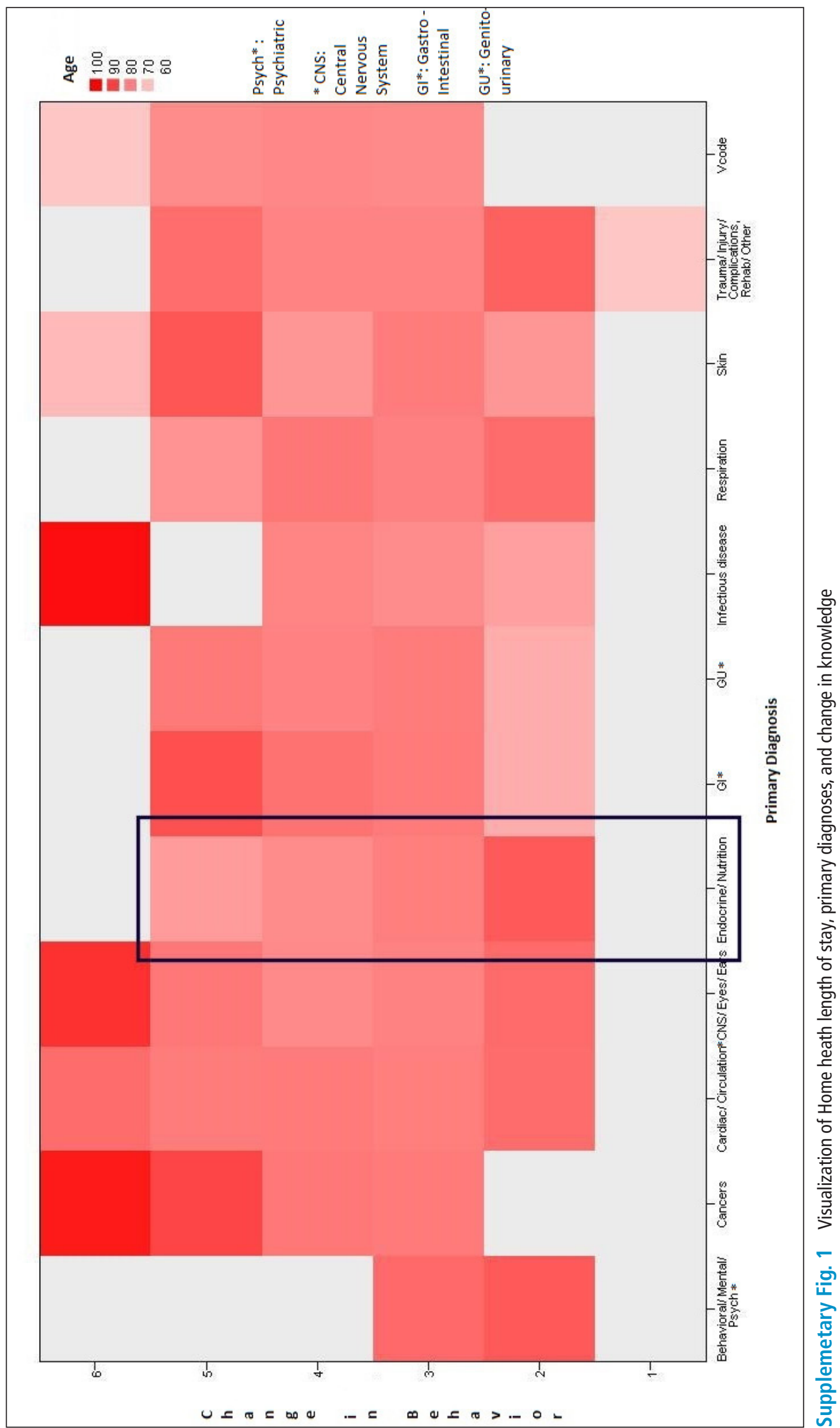




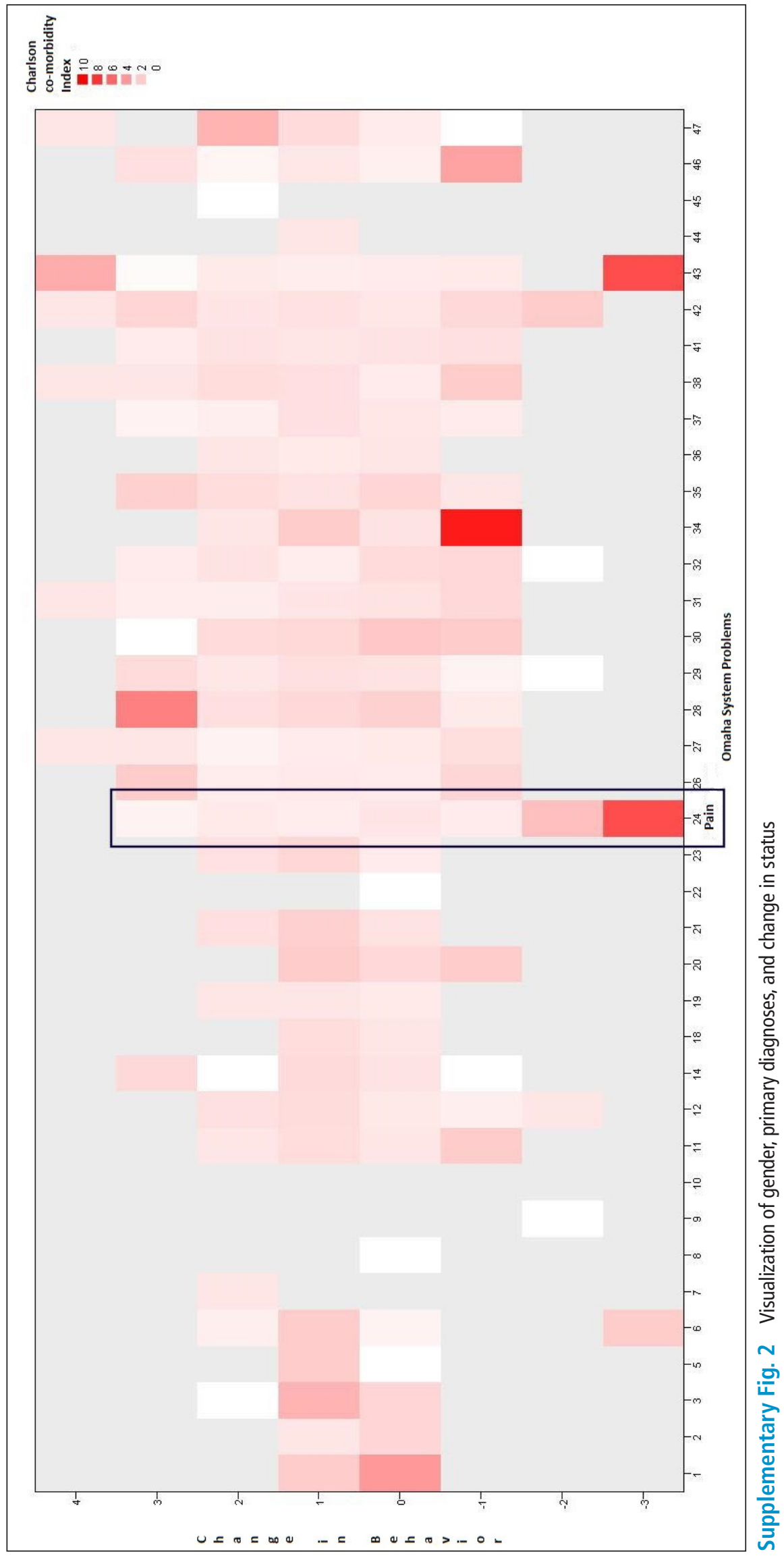


Supplementary Table 1 Grouping of 51 CCS categories to 11 disease groups

\begin{tabular}{|c|c|c|}
\hline Grouping & CCS Categories & CCS Categories (51 groups) \\
\hline \multirow[t]{4}{*}{ l) } & Infectious disease & \\
\hline & 2 & 1. 'Septicemia' \\
\hline & 5 & 2. 'HIV infectn' \\
\hline & $1,3,4,6,7,8,9,248,76,77$ & 3. Other Infections \\
\hline \multirow[t]{7}{*}{ II) } & Cancers & \\
\hline & 11,35 & 4. Head/ Neck/ Brain Ca \\
\hline & $12,13,14,15,17$ & 5. GI Cancer \\
\hline & 19 & 6. Lung Cancer \\
\hline & $24,25,26,27,28,29$ & 7. Sexual organ Cancer \\
\hline & $37,38,39$ & 8. Lymphomas / Leukemias \\
\hline & $21,23,32,41,42,43,44,45,47$ & 9. Other Cancers/ Tx/ Benign \\
\hline \multirow[t]{6}{*}{ III) } & Endocrine/ Nutrition & \\
\hline & 49 & 10. 'DiabMel no c' \\
\hline & 50 & 11. 'DiabMel w/cm' \\
\hline & 48,51 & 12. Other endocrine \\
\hline & $52,53,54,55,56,57,58$ & 13. 'Nutrit defic' \\
\hline & $59,60,62,63,64$ & 14. Anemia/ Blood disorders \\
\hline \multirow[t]{4}{*}{ IV) } & Behavioral/ Mental/ Psych & \\
\hline & $65,66,67,72$ & 15. Other Mental/ Emotional \\
\hline & 68 & 16. 'Senil/org mn' \\
\hline & 69,70 & 17. Major Psych \\
\hline \multirow[t]{7}{*}{ V) } & CNS/ Eyes/ Ears & \\
\hline & 82,79 & 18. 'Paralysis'/ Parkinsons \\
\hline & 83 & 19. 'Epilepsy/cnv' \\
\hline & $85,228,233$ & 20. Coma/brain dmg' \\
\hline & 89 & 21. 'Blindness' \\
\hline & $86,87,90,91,92,93,94$ & 22. Eye/ Ear \\
\hline & $95,216,81$ & 23. 'Other nervous $\mathrm{dx}$ ' \\
\hline \multirow[t]{9}{*}{ VI) } & Cardiac/ Circulation & \\
\hline & $97,98,99,111,112,113,117,120,121$ & 24. HTN \& Other circula. Dx \\
\hline & $100,101,102$ & 25. Ml \\
\hline & $103,104,96,213,245$ & 26. Other Heart Dx \\
\hline & 105,106 & 27. Conduction \\
\hline & 108 & 28. 'CHF;NONHP' \\
\hline & 109,110 & 29. 'Acute CVD' \\
\hline & $114,116,118,119$ & 30. 'Perip athero' \\
\hline & 115 & 31. 'Aneurysm' \\
\hline
\end{tabular}


Supplementary Table 1 Continued

\begin{tabular}{|c|c|c|}
\hline Grouping & CCS Categories & CCS Categories (51 groups) \\
\hline \multirow[t]{4}{*}{ VII) } & Respiration & \\
\hline & $122,123,125,126,129,130,133,134$ & 32. Other Respir \\
\hline & 127,128 & 33. 'COPD'/ asthma \\
\hline & 131 & 34. Adlt resp failure \\
\hline \multirow[t]{4}{*}{ VIII) } & GI & \\
\hline & $135,138,141,143,144,145,146,147,149$ & 35. Stomach/ Intest Dis \\
\hline & $150,151,152,153$ & 36. GI Disorders \\
\hline & $154,155,250,251,252$ & 37. Mild GI/ Fatigue \\
\hline \multirow[t]{5}{*}{$\mathrm{IX})$} & GU & \\
\hline & $156,157,158$ & 38. Renal \\
\hline & $159,160,161$ & 39. 'Ot dx kidney' \\
\hline & 162,163 & 40. 'Ot dx bladdr' \\
\hline & $164,165,166,167,168,170,172,175$ & 41. Genital/ reprod \\
\hline \multirow[t]{3}{*}{ X) } & Skin & \\
\hline & $197,198,200$ & 42. Other Skin \\
\hline & 199 & 43. 'Ulcer skin' \\
\hline \multirow[t]{9}{*}{$\mathrm{XI})$} & Trauma/ Injury/ Complications, Rehab/ Other & \\
\hline & 201, 202, 204, 205, 206, 207, 208, 209 & 44. Other Joint \\
\hline & 203 & 45. 'Osteoarthros' \\
\hline & $226,227,229,230,231$ & 46. Fractures \\
\hline & $211,212,225$ & 47. Other ortho \\
\hline & $232,234,235,236,237,239,240,244,248$ & 48. Wounds/ injuries \\
\hline & $237,238,242$ & 49. Complications \\
\hline & 254,257 & 50. Rehab/ Aftercare \\
\hline & 193, 195, 196, 217, 255, 259, 2607 & 51. Other \\
\hline
\end{tabular}

Supplementary Table 2 Diseases and Injuries Tabular Index
1. Infectious and parasitic diseases (001-139)
2. Neoplasms (140-239)
3. Endocrine, nutritional and metabolic diseases, and immunity disorders (240-279)
4. Diseases of the blood and blood-forming organs (280-289)
5. Mental disorders (290-319)
6. Diseases of the nervous system and sense organs (320-389)
7. Diseases of the circulatory system (390-459)
8. Diseases of the respiratory system (460-519)
9. Diseases of the digestive system (520-579)
10. Diseases of the genitourinary system (580-629)
11. Complications of pregnancy, childbirth, and the puerperium (630-679)
12. Diseases of the skin and subcutaneous tissue (680-709)
13. Diseases of the musculoskeletal system and connective tissue (710-739)
14. Congenital anomalies (740-759)
15. Certain conditions originating in the perinatal period (760-779)
16. Symptoms, signs, and ill-defined conditions (780-799)
17. Injury and poisoning (800-999) 


\section{References}

1. Home Health Providers. Centers for Medicare \& Medicaid Services web site. http://www.cms.gov/Medi care/Provider-Enrollment-and-Certification/CertificationandComplianc/HHAs.html. Updated April 9, 2013. Accessed February 5, 2014.

2. Henderson R. Employment outlook: 2010-2020: industry employment and output projections to 2020. Monthly Labor Rev 2012; 135(1): 65-83.

3. CY 2014 Home Health Prospective Payment System Rate Update, Conversion to ICD-10-CM, Home Health Quality Reporting Requirements, and Cost Allocation of Home Health Survey Expenses. Centers for Medicare \& Medicaid Services web site. http://www.cms.gov/Medicare/Medicare-Fee-for-Service-Pay ment/HomeHealthPPS/Home-Health-Prospective-Payment-System-Regulations-and-Notices-Items/ CMS-1450-P.html. Published July 3, 2013. Accessed September 27, 2014.

4. Bowles KH, Holland DE, Horowitz DA. A comparison of in-person home care, home care with telephone contact and home care with telemonitoring for disease management. J Telemed Telecare 2009; 15(7): 344-350.

5. Institute of Medicine. Capturing Social and Behavioral Domains in Electronic Health Records: Phase 1. Washington, DC: The National Academies Press; 2014.

6. Institute of Medicine. Capturing Social and Behavioral Domains and Measures in Electronic Health Records: Phase 2. Washington, DC: The National Academies Press; 2014.

7. Martin KS. The Omaha System: A Key to Practice, Documentation, and Information Management. 2nd ed. Omaha, NE: Health Connections Press; 2005.

8. Monsen KA, Peterson JJ, Mathiason MA, Kim E, Lee S, Chi CL, Pieczkiewicz DS. Data visualization techniques to showcase nursing care quality. Computers, Informatics, Nursing 2015; 33(10): 417-426.

9. Bui AAT, Aberle DR, Kangarloo H. Timeline: visualizing integrated patient records. Inf Technol Biomed 2007; 11(4): 462-473.

10. Monsen KA, Peterson JJ , Mathiason MA, Kim E, Lee S, Chi CL, Pieczkiewicz DS. Data visualization techniques to showcase nursing care quality. Comput Inform Nurs 2015; 33(10):417-426.

11. Torsvik T, Lillebo B, Mikkelsen G. Presentation of clinical laboratory results: an experimental comparison of four visualization techniques. J Am Med Inform Assoc 2013; 20(2): 325-331.

12. West VL, Borland D, Hammond WE. Innovative information visualization of electronic health record data: a systematic review. J Am Med Inform Assoc 2015; 22: 330-339.

13. Kim E, Monsen KA, Pieczkiewicz, D. Visualization of Omaha System data enables data-driven analysis of outcomes. Poster presented at the American Medical Informatics Association Annual Meeting; November 19, 2013; Washington, DC.

14. Votava B, Monsen KA. Visualization of patterns in public health nurse intervention data. Poster presented at the American Public Health Association Annual Meeting; November 16, 2014; New Orleans.

15. Westra BL, Oancea C, Savik K, Marek KD. The feasibility of integrating the Omaha System data across home care agencies and vendors. Comput Inform Nurs 2010; 28(3): 162-171.

16. Omaha System. The Omaha System: Solving the clinical data-information puzzle. http://omahasystem.org. Updated May 26, 2015.

17. Topaz M, Golfenshtein N, Bowles KH. The Omaha System: a systematic review of the recent literature. J Am Med Inform Assoc 2014; 21(1): 163-170.

18.2011 OASIS requirements in new and accredited HHAs seeking Medicare certification. Centers for Medicare \& Medicaid Services web site. https://www.cms.gov/Medicare/Quality-Initiatives-Patient-Assess ment-Instruments/OASIS/downloads/seekingmedicarecert.pdf. Published 2011. Accessed August 18, 2015.

19. Madigan EA, Fortinsky RH. Interrater reliability of the outcomes and assessment information set: results from the field. Gerontologist 2004; 44(5): 689-692.

20. Riggs JS, Madigan EA, Fortinsky RH. Home health care nursing visit intensity and heart failure patient outcomes. Home Health Care Manag Pract 2011; 23(6): 412-420.

21.Diseases and injuries tabular index. ICD-9-CM web site. http://icd9cm.chrisendres.com/index.php?action=contents. Published October 1, 2008. Accessed March 15, 2015.

22. Wilkinson L, Friendly M. The History of the Cluster Heat Map. The American Statistician 2009; 63(2): 179-184.

23. Kirk A. Data Visualization: A Successful Design Process. Birmingham, UK: Packt Publishing; 2012.

24. Gehlenborg N, Wong B. Points of view: heat maps. Nat Methods 2012; 9(3): 213-213.

25. Hu TW, Wagner TH, Bentkover JD, Leblanc K, Zhou SZ, Hunt T. Costs of urinary incontinence and overactive bladder in the United States: a comparative study. Urology 2004; 63(3): 461-465. 
26. John G, Gerstel E, Jung M, Dallenbach P, Faltin D, Petoud V, Zumwald C, Rutschmann OT. Urinary incontinence as a marker of higher mortality in patients receiving home care services. BJU Int 2014; 113(1): $113-119$.

27. Westra BL, Bliss DZ, Savik K, Hou Y, Borchert A. Effectiveness of wound, ostomy, and continence nurses on agency-level wound and incontinence outcomes in home care. Home Healthc Nurse 2014; 32(2): 119-127.

28. Sims J, Browning C, Lundgren-Lindquist B, Kendig H. Urinary incontinence in a community sample of older adults: prevalence and impact on quality of life. Disabil Rehabil 2011; 33(15-16): 1389-1398.

29. Du YF, Ou HY, Beverly EA, Chiu CJ. Achieving glycemic control in elderly patients with type 2 diabetes: a critical comparison of current options. Clin Interv Aging 2014; 9: 1963-1980.

30.Eckerblad J, Theander K, Ekdahl A, Unosson M, Wirehn A, Milberg A, Krevers B, Jaarsma T. Symptom burden in community-dwelling older people with multimorbidity: a cross-sectional study. BMC Geriatr 2015; 15: 1.

31. Morrissey MB, Viola D, Shi Q. Relationship between pain and chronic illness among seriously ill older adults: expanding role for palliative social work. J Soc Work End Life Palliat Care 2014; 10(1): 8-33.

32. Institute of Medicine. Relieving Pain in America: A Blueprint for Transforming Prevention, Care, Education, and Research: 2011, National Academies Press: Washington (DC).

33. American Geriatrics Society. Pharmacological management of persistent pain in older persons. J Amer Geri Soc 2009; 57(8):1331-1346.

34.Barber JB, Gibson SJ. Treatment of chronic non-malignant pain in the elderly: Safety considerations. Drug Safety 2009; 32(6): 457-474. 